Article

\title{
An Analysis of Starved EHL Point Contacts with Reflow
}

\author{
Takashi Nogi \\ Aerospace Research and Development Directorate, Japan Aerospace Exploration Agency \\ 7-44-1 Jindaiji-higashimachi, Chofu, Tokyo 182-8522, Japan \\ Corresponding author: nogi.takashi@jaxa.jp
}

\begin{abstract}
( Manuscript received 7 December 2014; accepted 6 January 2015; published 28 February 2015 )
( Reprinted from JOURNAL OF JAPANESE SOCIETY OF TRIBOLOGISTS, 59, 4, 2014, 239-250; Original manuscript received 4 October 2013 )

In the starved lubrication regime, the film thickness can be much less than the fully flooded film thickness. The film thickness reduction is related to the inlet meniscus distance. The inlet meniscus and the film thickness can be computed for a given inlet film thickness distribution which is generally non-uniform. The inlet film thickness distribution, which is essentially the outlet film thickness distribution of the previous contact, is affected by the surface tension. In this paper, a modified Coyne-Elrod boundary condition is used to calculate the inlet film thickness distribution, taking the surface tension into account. A numerical analysis of EHL point contacts is carried out and solutions of the inlet meniscus and the film thickness are presented. At lower capillary numbers, side bands formed by reflow in the outlet region can replenish the contact owing to reflow in the inlet region. At higher capillary numbers, the side bands move away from the center of the track and the inlet distance decreases. This is a main cause of starvation in EHL point contacts. A good agreement is found between the numerical solutions and experimental results.
\end{abstract}

Keywords: starved EHL, point contact, reflow, side band, capillary number, amount of oil

\section{Introduction}

It is important to predict the film thickness in starved EHL contacts in order to ensure the film thickness larger than the surface roughness and prevent surface damage due to asperity contacts. Wedeven et al. [1] related the film thickness decrease in starved EHL point contacts to the inlet meniscus distance. Hamrock and Dowson (H-D) [2] proposed formulas for the starved EHL film thickness as a function of the inlet meniscus distance ("inlet distance"). However, the usability of such formulas is limited since the inlet distance is unknown in real applications including rolling bearings. Chevalier et al. [3] applied the Elrod [4] algorithm for cavitation analysis to EHL problems and showed that if the thickness of oil layers present on the surfaces ("initial film thickness," a parameter representing the amount of oil) is known, the inlet meniscus can be obtained numerically. They investigated the film thickness decay rate due to side leakage by repeatedly using the outlet film thickness behind the contact as the initial film thickness. In such an analysis, however, the film thickness monotonically decreases and hence the transition from the fully flooded regime to the starved regime cannot be simulated.

According to Kingsbury [5], the degree of starvation is determined by the balance between oil loss due to side leakage and replenishment to the inlet due to some mechanism. Chiu [6] proposed a replenishment model in which the outlet film thickness at the rear of the contact is approximated by the Hertzian profile, and is flattened between contacts due to surface tension.

Cann et al. [7] experimentally investigated the effect of operating conditions on the starved EHL film thickness and proposed a parameter $\mathrm{SD}=\eta_{0} u a / h_{i} \gamma$ which governs the degree of starvation. $h_{i}$ is defined as "the amount of oil present in the vicinity of the track." Since it is calculated as the volume of the applied oil divided by the width and the length of the track, it is regarded as the initial film thickness in the present study. It was pointed out that the replenishment is very slow between contacts and occurs mostly around the contact, though its mechanism was not investigated analytically.

Chevalier et al. [3] showed that in experiments under repeated overrollings, the center of the contact can be heavily starved while the sides of the contact can remain nearly fully flooded. This phenomenon can be simulated numerically using a non-uniform inlet film thickness which is thin at the center and thick at the sides of the track. However, the inlet film thickness used in [3] was only a function of $Y$ and the effect of operating conditions was not considered.

Shibasaki et al. [8,9] proposed a numerical method for coupled simulation of starved EHL and macroscopic 
flow. The inlet film thickness in ball bearings was obtained from the oil feed rate. Ball-on-disk tests were simulated by empirically implementing the effect of the flow around the contact.

According to an observation by Pemberton and Cameron (P-C) [10], unless the rolling speed is extremely low, the flow having passed around the contact does not merge in the outlet region and splits into two "side bands" on both sides of the track. The side bands are forced to spread laterally in the converging region because the sum of the film thickness on two surfaces is larger than the gap height at either side of the contact. The majority of the oil flows around the contact, but the remainder replenishes the inlet to form an equilibrium with oil loss due to side leakage. It is important that flow from the sides of the meniscus toward the center of the track occurs in both the inlet and the outlet region. This is called "reflow" in this paper.

In the aforementioned equilibrium, the inlet film thickness is nearly equal to the outlet film thickness in the previous contact and the nonuniform inlet film thickness including the side bands results from the reflow in the outlet region. Surface tension at the film rupture point is likely to affect this reflow, but conventional boundary conditions for EHL problems do not take surface tension into account. In the Elrod algorithm, the pressure gradient is zero at the film rupture point in the outlet region and the film thickness at downstream is equal to the gap height at the film rupture point (see Fig. 3.4 in [11]), though the pressure gradient and the film thickness are discontinuous at the film formation point in the inlet region. That is, the boundary condition used in the outlet region is the Reynolds condition neglecting surface tension. On the other hand, the Coyne and Elrod (C-E) [12,13] boundary condition is known to take surface tension into account. According to the C-E condition, the film thickness at downstream is less than the gap height at the film rupture point, and the ratio of the two is determined by the capillary number.

This paper presents an analysis of starved EHL point contacts with reflow. First, based on the C-E boundary condition determined by the capillary number, a method is proposed to calculate the nonuniform inlet film thickness including the side bands. This inlet film thickness is used with the numerical algorithm by Chevalier et al. and it is clarified how the dimensionless initial film thickness and the capillary number affect the starved EHL film thickness. In addition, a comparison with experimental results validates the present analysis for the shape of the inlet meniscus and the reduction factor of the central film thickness.

\section{Analytical methods}

\subsection{Numerical solution}

In this study, point contacts with $R_{x}=R_{y}$ (circular contacts) are considered. The numerical algorithm by
Chevalier et al. [3] solves the dimensionless Reynolds equation including a parameter $\theta$, the ratio of the film thickness to the gap height, by using the multigrid method. The dimensionless Reynolds equation is given by

$$
\frac{\partial}{\partial X}\left(\frac{\bar{\rho} H^{3}}{\bar{\eta}} \frac{\partial P}{\partial X}\right)+\frac{\partial}{\partial Y}\left(\frac{\bar{\rho} H^{3}}{\bar{\eta}} \frac{\partial P}{\partial Y}\right)=\lambda \frac{\partial(\bar{\rho} \theta H)}{\partial X}
$$

The dimensionless gap height is given by

$$
\begin{aligned}
& H(X, Y)=H_{0}+ \\
& \frac{X^{2}}{2}+\frac{Y^{2}}{2}+\frac{2}{\pi^{2}} \int_{-Y_{0}}^{Y_{0}} \int_{-X_{0}}^{X_{0}} \frac{P\left(X^{\prime}, Y^{\prime}\right) \mathrm{d} X^{\prime} \mathrm{d} Y^{\prime}}{\sqrt{\left(X-X^{\prime}\right)^{2}+\left(Y-Y^{\prime}\right)^{2}}}
\end{aligned}
$$

The film thickness is given by $\theta H$. The film thickness in the film rupture region is the sum of the thickness of two oil layers sticking to the surfaces. The force balance equation is given by

$$
\int_{-Y_{0}}^{Y_{0}} \int_{-X_{0}}^{X_{0}} P(X, Y) \mathrm{d} X \mathrm{~d} Y=\frac{2 \pi}{3}
$$

Equations (1)-(3) are solved with the following boundary conditions:

$$
\begin{aligned}
& P\left(-X_{0}, Y\right)=P\left(X_{0}, Y\right)=P\left(X,-Y_{0}\right)=P\left(X, Y_{0}\right)=0 \\
& \theta\left(-X_{0}, Y\right)=H_{\text {oil }}(Y) / H\left(-X_{0}, Y\right)
\end{aligned}
$$

We use the viscosity-pressure relation proposed by Roelands:

$$
\begin{aligned}
& \bar{\eta}(P)=\exp \left(p_{r} \alpha / z\left(-1+\left(P p_{h} / p_{r}\right)^{z}\right)\right) \\
& p_{r} \alpha / z=\ln \left(\eta_{0}\right)+9.67
\end{aligned}
$$

and the density-pressure relation proposed by Dowson and Higginson:

$$
\bar{\rho}(P)=\frac{0.59 \times 10^{9}+1.34 P p_{h}}{0.59 \times 10^{9}+P p_{h}}
$$

The boundary between the pressurized region and the film rupture region is automatically determined in the process of relaxation. If $P<0$ on a grid point in the pressurized region $(\theta=1)$, let $P=0$ and $\theta$ is recalculated so that the flow rate is continuous across the boundary, while if $\theta>1$ on a grid point in the film rupture region $(P$ $=0$ ), let $\theta=1$ and $P$ is recalculated [11]. The Elrod algorithm characteristically applies this procedure to the inlet region as well as the outlet region to determine the boundary. Therefore, it is difficult to allow negative pressure only in the outlet region.

Elastic deformation is computed by the FFT method [14]. In [14], the domain was extended with $P=0$ to reduce numerical errors due to false periodicity. In the present study, however, this is omitted because the high pressure Hertzian contact is much smaller than the domain size that must contain the entire meniscus.

\subsection{C-E theory}

C-E $[12,13]$ solved the Navier-Stokes equations for two-dimensional flow, taking surface tension into account and assuming that the velocity profile is quadratic. The pressure flow in the direction opposite to the shear flow is significant in the vicinity of the film 


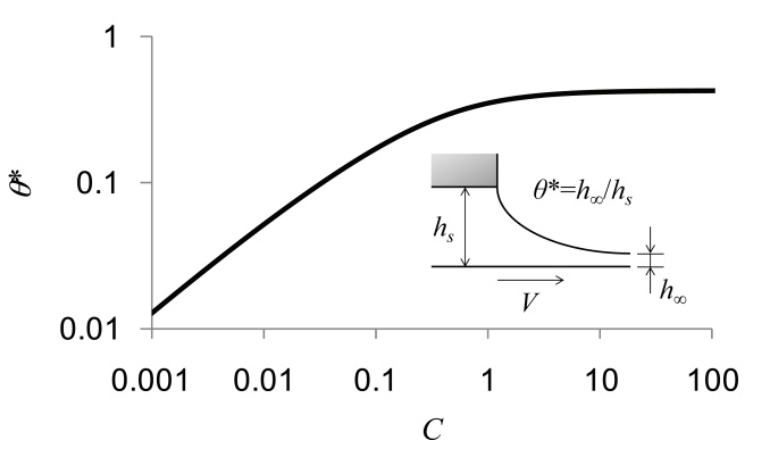

Fig. 1 COYNE-ELROD boundary condition

rupture point, while the shear flow is dominant at downstream from the film rupture point. Thus the film thickness decreases from the film rupture point to downstream due to the continuity of the flow rate. $\theta^{*}$ is calculated as a function of the capillary number defined by

$$
C=\eta_{0} v / \gamma
$$

Figure 1 shows calculated results of $\theta^{*}$. It was pointed out that the $\mathrm{C}$-E theory is computationally unstable when reverse flow occurs [15]. Therefore, in this study, the C-E theory is modified so that the velocity gradient on the free surface becomes zero, as described in the Appendix A.

This study assumes pure rolling contacts with the slide/roll ratio of zero (below simply referred to as rolling contacts). We can consider applying the C-E theory to rolling contacts, even though it assumes sliding contacts. It seems reasonable to use $\theta^{*}$ in rolling contacts if we assume that the meniscus is symmetric about the centerline of the film thickness and that $h_{\infty}$, the film thickness at downstream is equal on two surfaces, where the gap at the film rupture point is defined as $2 h_{s}$. Dalmaz [16] investigated the relation between the film rupture point and the capillary number in line contacts. The film rupture point predicted by the C-E theory was shown to agree with experimental results in sliding contacts. However, experimental results in rolling contacts were not compared with the C-E theory. Therefore, $\theta^{\prime}$ is calculated from the experimental results in [16], which is the ratio of the film thickness at downstream to the gap height at the film rupture point in rolling contacts, as described in the Appendix B. The film thickness in the film rupture region is calculated by using the Birkhoff and Hays [17] equation. The following equation is obtained.

$$
\theta^{\prime}=1.2 \theta^{*}
$$

In this study, the above equation is used to obtain the boundary of the cavity (film rupture region in the inner part of the track in the outlet region).

\subsection{Case of no reflow}

In this section, we consider the case in which the reflow in the outlet region can be neglected. The cavity

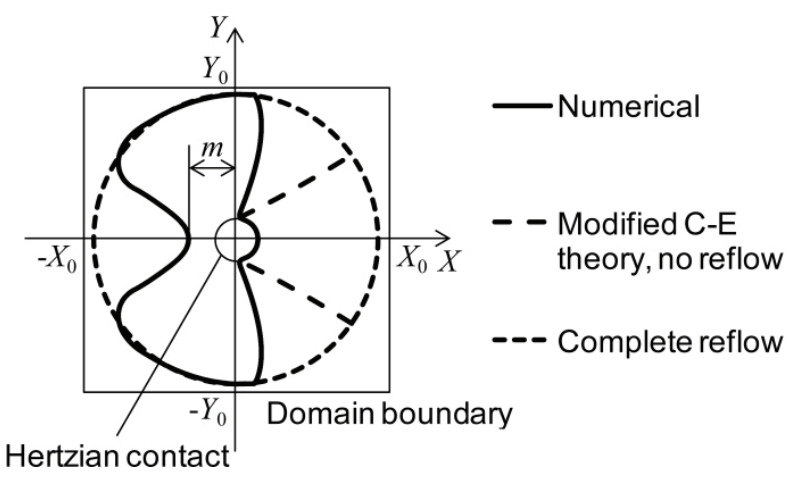

Fig. 2 Meniscus and cavity boundary

boundary in this case is applied to the inner part of the track (see the section 2.5). A solid line in Fig. 2 shows an example of numerical results of the meniscus shape. In this case, the outlet film thickness is obtained as shown by a dashed line in Fig. 3. This is a solution in the case of no reflow in the outlet region since it is computed without negative pressure. According to the Reynolds condition, the film thickness at downstream is equal to the gap height at the film rupture point. Since the film rupture point is close to the position of the minimum gap, the outlet film thickness is approximately of the Hertzian profile.

On the other hand, experiments show that the cavity boundary is approximately straight and extends obliquely downstream from the Hertzian circle. For example, in Fig. 3.15 in [18], we can see that the angle between the boundary and the $X$ axis is $35^{\circ}$, which is very different from numerical solutions without surface tension taken into account. Therefore, in this study, the cavity boundary is obtained based on the C-E theory. Since this boundary is applied to the inner part of the track where the effect of the reflow is supposed to be small, we assume that the numerical solution can be used as for the outlet film thickness, which means that the film thickness in the cavity in the outlet region is given by $\theta\left(X_{0}, Y\right) H\left(X_{0}, Y\right)$. Applying the $\mathrm{C}$-E condition to the film rupture point, the following equation is obtained.

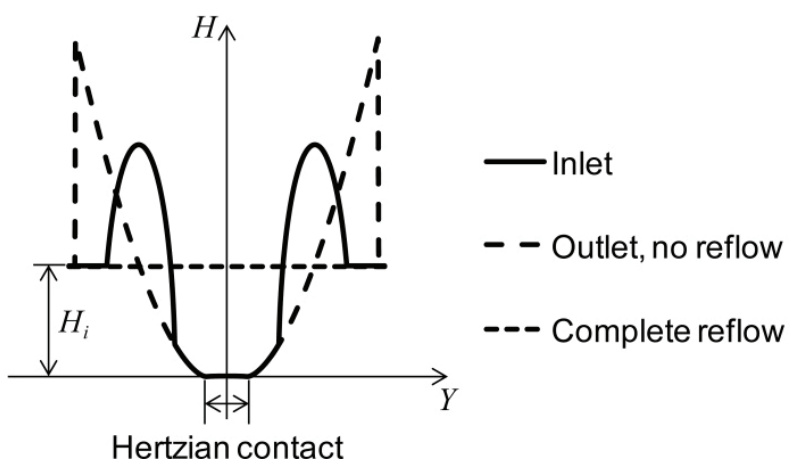

Fig. 3 Inlet and outlet film thickness 


$$
\theta\left(X_{0}, Y\right) H\left(X_{0}, Y\right)=\theta^{\prime} H(X, Y)
$$

Generally, cavitation is classified into three categories: (1) flow separation at the film rupture point, (2) gas (air or vapor) diffusion out of the oil, and (3) bubbles formed upstream and carried downstream without enough growth to alter the flow field [19]. The C-E theory assumes (1), but in the vicinity of the boundary, microscopic bubbles and streamers are observed $[1,10]$. Therefore, the physical state of the cavity boundary is rather close to (2) or (3), and the C-E theory assuming (1) is not likely to hold rigorously.

Inside the Hertzian track $(|Y|<1)$, the cavity boundary in the numerical solution approximately coincides with the Hertzian circle [3]. This generally agrees with experimental observations such as Fig. 3.15 in [18]. However, the boundary by the $\mathrm{C}$-E condition is at a downstream location compared with the numerical solution by the Reynolds condition [19]. Thus we assume that the numerical solution can be used as for the boundary inside the Hertzian track.

Assuming that the bubbles and the streamers flow downstream along the boundary given by Eq. (9), we consider a boundary that is parallel to Eq. (9) and matches the numerical solution at $|Y|=1$ :

$$
\theta\left(X_{0}, Y\right) H\left(X_{0}, Y\right)=\theta^{\prime} H(X+\delta, Y)
$$

We find $X_{a}$ that satisfies $\theta\left(X_{a}, Y_{a}\right)=1$ and $\theta\left(X_{a}+\Delta_{X}, Y_{a}\right)<$ 1 in the numerical solution, where $Y_{a}$ is the $Y$ coordinate closest to 1 on the grid points, and solve $\theta\left(X_{0}, Y_{a}\right) H\left(X_{0}, Y_{a}\right)$ $=\theta^{\prime} H\left(X_{b}, Y_{a}\right)$ for $X_{b}$ to calculate $\delta=X_{b}-X_{a}$, where the film thickness between the grid points is calculated by linear interpolation. In this study, since the solution is symmetric about the $X$ axis, $\delta$ is obtained only in the region where $Y>0$.

C-E suggest that the surface velocity component normal to the boundary, $v=\mathbf{u} \cdot \mathbf{n}$ should be used in Eq. (7), where $\mathbf{u}$ and $\mathbf{n}$ are the surface velocity and the unit vector normal to the boundary, respectively [13]. In this study, however, considering the existence of the aforementioned bubbles and streamers, the boundary at the film rupture point is assumed to be locally normal to the $X$ axis and the velocity in the $X$ direction is used ( $v=$ $u$ ). Therefore, $\theta^{\prime}$ is only a function of $C$. A dashed line in Fig. 2 shows an example of the boundary obtained as described above. The shape of this cavity boundary is similar to that observed experimentally $[1,10]$. The straight boundary is observed when the speed of the above bubbles is higher than that of photography. In other words, the straight boundary is the envelope of the air/oil interface of a number of bubbles.

\subsection{Case of complete reflow}

In this section, we consider the case in which the effect of the reflow in the outlet region is dominant. The meniscus shape in this case is applied to the outer parts of the track (see the section 2.5). Although the numerical solution of the outlet meniscus is as shown by the solid line in Fig. 2, the actual meniscus in the outer parts of the track is close to elliptical and hence symmetric between the inlet and the outlet region [10]. Therefore, in this study, the meniscus is approximated by an ellipse given by

$$
\frac{X^{2}}{r_{X}^{2}}+\frac{Y^{2}}{r_{Y}^{2}}=1
$$

$r_{X}$ and $r_{Y}$ are obtained from the numerical solution for $H_{o i l}(Y)=H_{i} . r_{X}$ is obtained from $\theta\left(-r_{X}-\Delta_{X}, 0\right)<1$ and $\theta\left(-r_{X}, 0\right)=1 . r_{Y}$ is obtained from $\theta\left(0, r_{Y}\right)=1$ and $\theta\left(0, r_{Y}\right.$ $\left.+\Delta_{Y}\right)<1$ (in the region where $Y>0$ due to symmetry). In this case, the inlet meniscus becomes almost circular [3].

Equation (11) is applied to the outer parts of the track, where the film thickness is virtually symmetric between the converging and the diverging region. Therefore, if we assume that the negative pressure in the outlet region is symmetric to the positive pressure in the inlet region, the gap height and the pressure gradient on the inlet boundary are equal to those on the outlet boundary, and hence the inlet film thickness is equal to the outlet film thickness. Thus, as shown by a dotted line in Fig. 3, we assume that the outlet film thickness is uniform: $H_{o i l}(Y)=$ $H_{i}$.

$r_{X}$ is fixed but $r_{Y}$ is modified each time it changes in iterations. A dotted line in Fig. 2 shows an example of the meniscus obtained as described above.

\subsection{Side bands}

In repeated overrollings, the center of the contact can be starved while the contact sides can remain fully flooded [3]. Since this is in between the two extreme cases of no reflow in the section 2.3 and complete reflow in the section 2.4 , it is reasonable to apply the solution in the case of no reflow and complete reflow to the inner part and the outer parts of the track, respectively. Thus the outlet film thickness is given by

$$
\begin{aligned}
& H_{e}(Y)=\theta\left(X_{0}, Y\right) H\left(X_{0}, Y\right) ;|Y|<Y_{1} \\
& H_{e}(Y)=H_{i} ; Y_{1} \leq|Y|
\end{aligned}
$$

where $Y_{1}$ is the $Y$ coordinate of the intersection point of the boundary in the case of no reflow and the meniscus in the case of complete reflow (let $Y_{1}>0$ due to symmetry). In order to find $Y_{1}, X_{A}(Y)$, the solution of Eq. (10) and $X_{B}(Y)$, the solution of Eq. (11) are obtained in the outlet region (the film thickness between the grid points are calculated by linear interpolation). Then the intersection point of $X_{A}(Y)$ and $X_{B}(Y)$ are obtained (the boundary between the grid points are calculated by linear interpolation).

When the outlet film thickness is determined as described above, the flow rate is discontinuous from the inlet to the outlet. Namely, since the outlet film thickness is equal to the constant inlet film thickness $H_{i}$ in the outer parts of the track and less than $H_{i}$ in the inner part of the track, the flow rate in the outlet region is less than in the inlet region. Actually, however, thick oil layers are formed on both sides of the track, corresponding to the difference in the flow rate between the inlet and the outlet, 


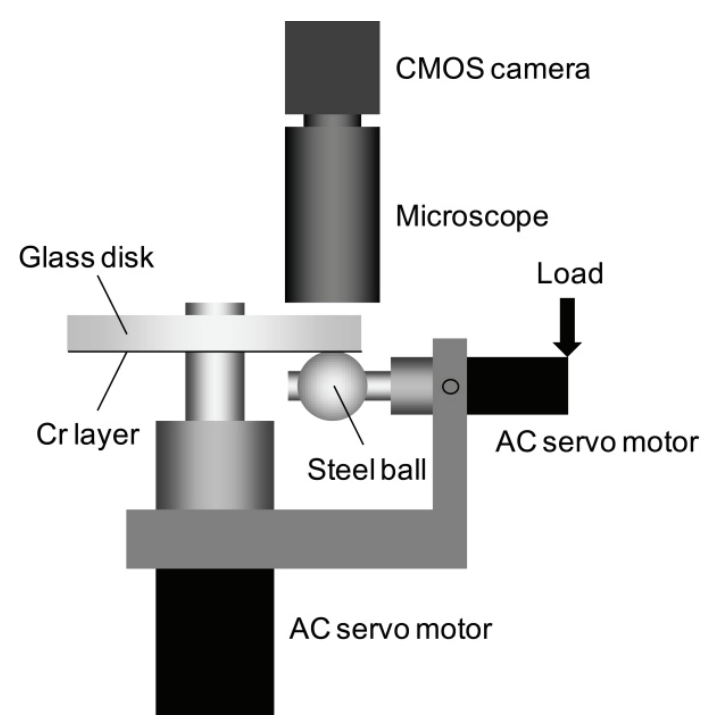

Fig. 4 Schematic diagram of test rig

and the continuity equation is satisfied. These are the "side bands". In this study, the replenishment between contacts is assumed to be negligible and the sum of the outlet film thickness given by Eq. (12) and the side bands is used as the inlet film thickness. The distance between the center of each band and the center of the track is assumed to be $Y_{1}$.

Popovici [18] estimated the thickness of an oil layer present on a disk surface from interferometric images in front of the film formation point. In Fig. $4.23(0.06 \mathrm{~m} / \mathrm{s})$ in [18], the profile of side bands is close to elliptical. Thus the inlet film thickness is approximated by

$$
H_{o i l}(Y)=\max \left(H_{s b}\left(1-\left(\frac{|Y|-Y_{1}}{W_{s b}}\right)^{2}\right)^{1 / 2}, H_{e}(Y)\right)
$$

From the continuity of the flow rate,

$$
\int_{-Y_{0}}^{Y_{0}} H_{o i l}(Y) d Y=2 Y_{0} H_{i}
$$

In Fig. $4.23(0.06 \mathrm{~m} / \mathrm{s})$ in [18], the dimensionless meniscus radius $r_{Y}=3.0$. The numerical solution of the same conditions is obtained with $H_{i}=1.0$, which gives $H_{1}=3.33$, where $H_{1}$ is $H$ at the intersection point of Eqs. (10) and (11). The maximum film thickness sticking to the disk surface is $2.3 \mu \mathrm{m}$ in Fig. $4.23(0.06 \mathrm{~m} / \mathrm{s})$ in [18] and if we assume that the film thickness on the two surfaces is equal, the sum of the film thickness on the two surfaces is $4.6 \mu \mathrm{m}$. This corresponds to $H_{s b}=2.4$. In this study, we assume that $H_{s b}$ is proportional to $H_{1}$ with a constant coefficient. From the above value of $H_{1}$ and $H_{s b}$,

$$
H_{s b}=0.72 H_{1}
$$

If we calculate $H_{s b}$ from Eq. (15) and numerically solve Eq. (14) for $W_{s b}$, the inlet film thickness is determined from Eq. (13). Equation (14) is solved by the Newton-Raphson method expressed by

$$
\begin{aligned}
& f(Y)=\int_{-Y_{0}}^{Y_{0}} H_{o i l} d Y-2 Y_{0} H_{i} \\
& W_{s b}-\frac{f}{d f / d Y} \rightarrow W_{s b}
\end{aligned}
$$

A solid line in Fig. 3 shows an example of the inlet film thickness obtained as described above.

The numerical solution of Eqs. (1)-(4) and the calculation of Eq. (13) for the inlet film thickness were iterated one after the other. In this study, $1025 \times 1025$ grid points were used. The iteration was started from the

Table 1 Experimental conditions

\begin{tabular}{c|c|c|c|c|c|c}
\hline$\#$ & $\begin{array}{c}\gamma \\
\mathrm{N} / \mathrm{m}\end{array}$ & $\begin{array}{c}\eta_{0} @ 20^{\circ} \mathrm{C} \\
\mathrm{Pa} \cdot \mathrm{s}\end{array}$ & $\begin{array}{c}\alpha \\
/ \mathrm{GPa}\end{array}$ & $\begin{array}{c}u \\
\mathrm{~m} / \mathrm{s}\end{array}$ & $\begin{array}{c}w \\
\mathrm{~N}\end{array}$ & $\begin{array}{c}h_{i} \\
\mu \mathrm{m}\end{array}$ \\
\hline 1 & 0.03 & 0.093 & 24.0 & $0.1,0.2,0.5$ & 20 & 34 \\
\hline 2 & 0.03 & 0.093 & 24.0 & $0.1,0.2,0.5$ & 20 & 19 \\
\hline 3 & 0.03 & 0.093 & 24.0 & $0.1,0.2,0.5$ & 10 & 22 \\
\hline 4 & 0.03 & 0.093 & 24.0 & $0.1,0.2$ & 20 & 13 \\
\hline 5 & 0.031 & 0.25 & 19.0 & $0.05,0.1,0.2$ & 20 & 34 \\
\hline 6 & 0.031 & 0.25 & 19.0 & $0.05,0.1,0.2$ & 10 & 34 \\
\hline 7 & 0.031 & 0.25 & 19.0 & $0.05,0.1$ & 20 & 22 \\
\hline 8 & 0.021 & 0.52 & 20.0 & 0.05 & 20 & 41 \\
\hline 9 & 0.021 & 0.52 & 20.0 & 0.05 & 10 & 63 \\
\hline 10 & 0.021 & 0.52 & 20.0 & 0.05 & 20 & 30 \\
\hline
\end{tabular}


inlet film thickness $H_{\text {oil }}(Y)=H_{i}$. In most cases, the solution converged in a few iterations.

\section{Experimental methods}

Using a ball-on-disk test rig, a borosilicate glass disk (BK7, Young modulus $E=70 \mathrm{GPa}$, Poisson ratio $v=0.2$ ) with a $\mathrm{Cr}$ layer on the contact surface and a steel ball (SUS440C, $E=200 \mathrm{GPa}, v=0.3$ ) of $25.4 \mathrm{~mm}$ in diameter, the meniscus was observed and the film thickness was measured by interferometry. Figure 4 and Table 1 show a schematic diagram of the test rig and experimental conditions, respectively. The slide/roll ratio was set to zero and a small amount of oil was applied to the contact. Paraffinic mineral, synthetic hydrocarbon and perfluoropolyether oils were used for \#1-4, \#5-7 and \#8-10 in Table 1, respectively. $h_{i}$ is difficult to measure and in [7], it was calculated as the volume of the applied oil divided by the width and the length of the track. In this study, $h_{i}$ was determined so that $r_{Y}$ is consistent between the numerical and the experimental results in the case of the lowest speed for each oil. Oblique light sources were used and the meniscus shape was observed

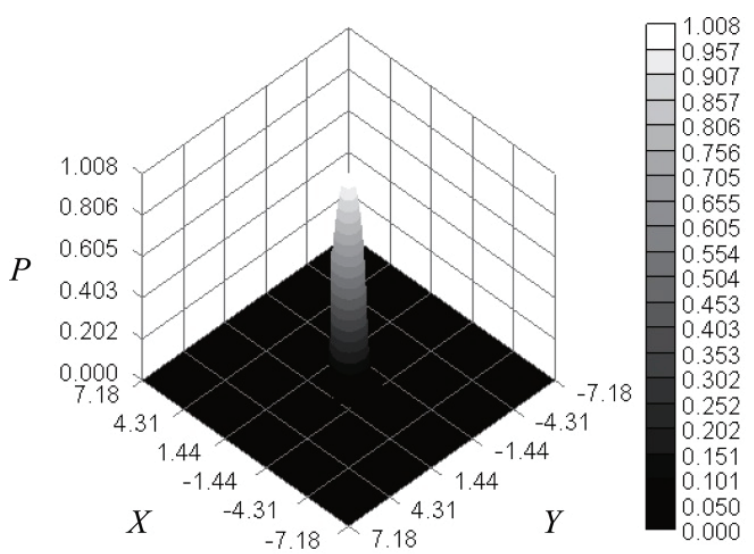

(a) Pressure

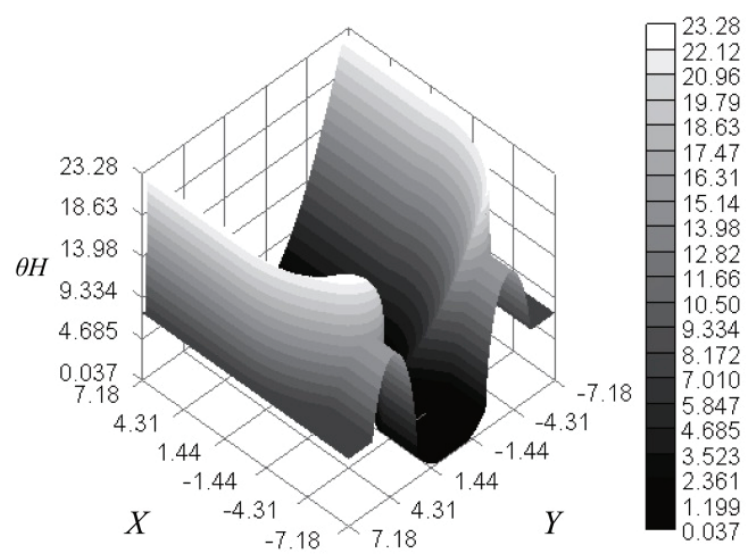

(b) Film thickness

Fig. 5 Dimensionless pressure and film thickness $(\# 4, u=0.1 \mathrm{~m} / \mathrm{s})$ in a similar manner to P-C's experiment [10].

Experimental methods described above were similar to those in [18], but a silica spacer layer was not used. In addition, calibration by using the clearance around a static Hertzian contact was difficult due to low magnification of the microscope needed to capture a wide area containing the whole meniscus. Therefore, interference images under fully flooded conditions were preliminarily taken, changing the speed at a load of $20 \mathrm{~N}$ for each oil, and $u_{r e f}$, the speed under fully flooded conditions was found that minimizes the color difference (the distance in the RGB color space) between the fully flooded and the starved regime. Since the central film thickness is proportional to the rolling speed to the power of 0.67 and the load to the power of -0.067 according to the fully flooded H-D formula [2], the reduction factor of the central film thickness is calculated by

$$
\frac{h_{c}}{h_{c f f}}=\left(\frac{u_{r e f}}{u}\right)^{0.67}\left(\frac{20}{w}\right)^{-0.067}
$$

where the unit of $w$ is $\mathrm{N}$.
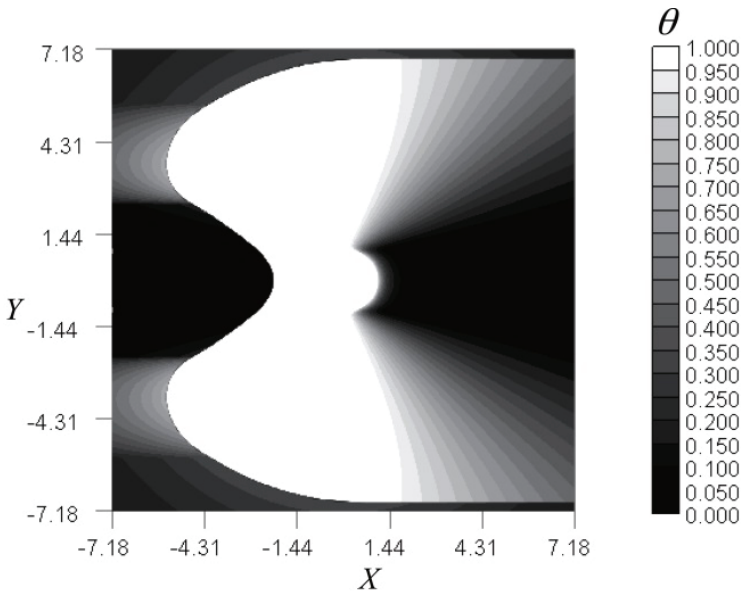

(a) Filling ratio

$Q$

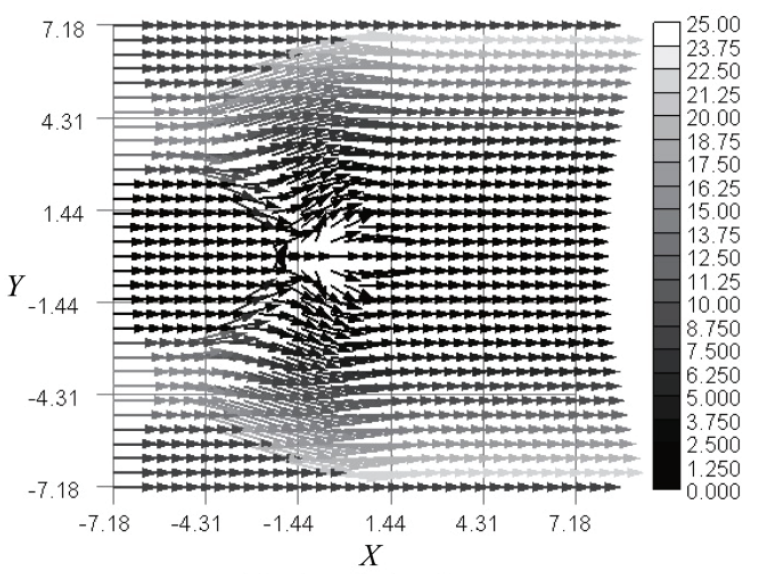

(b) Dimensionless flowrate

Fig. 6 Filling ratio and dimensionless flow rate (\#4, $u=0.1 \mathrm{~m} / \mathrm{s})$ 


\section{Results}

For the case \#4 $(u=0.1 \mathrm{~m} / \mathrm{s})$ in Table 1 , computed results of the pressure and the film thickness are shown in Fig. 5 (a) and (b), respectively. The pressure outside the contact is slightly different from that in the fully flooded regime but compared with the Hertzian pressure, it is too small to be clearly seen in Fig. 5 (a). As seen in Fig. 5 (b), the amount of oil at the center of the track is much less than that at the sides of the track. Where $X>0$, the EHL film thickness is approximately of the Hertzian profile since the reflow in the outlet region is not considered in the numerical solution. This is not realistic as described above, though the reflow is considered in the calculation of the inlet film thickness.

Figure 6 (a) and (b) show the ratio of the film thickness to the gap height and the dimensionless flow rate per unit width. In Fig. 6 (a), the region where $\theta=1$ is pressurized and the inlet meniscus shows a butterfly shape, which is typical in starved EHL contacts [10]. It can be seen in Fig. 5 (b) and Fig. 6 (b) that the film thickness of the side bands is larger than the gap height at either side of the contact. Therefore, the oil is forced to spread laterally in the converging region. The majority of the flow passes around the contact, but the remainder replenishes the inlet. This replenishment and oil loss due to side leakage are in equilibrium [10].

Figures 7, 8 and 9 show calculated results of the inlet film thickness for \#2, 3 and 4 in Table 1, respectively. These figures show that the dimensionless maximum film thickness of the side bands is nearly proportional to the dimensionless initial film thickness. As explained later, the side bands move away from the center of the track as the speed increases.

Figures 10, 11 and 12 show analytical results and photographs of the meniscus for \#2, 3 and 4 in Table 1, respectively. In the same manner as in Fig. 2, a solid line shows the numerical solution while the dashed and the dotted lines show Eqs. (10) and (11), respectively.

In the inlet region, the numerical solution of the meniscus agrees well with the experimental results. The meniscus at the sides of the track where the inlet film thickness is large agrees well with Eq. (11), while the meniscus at the center of the track where the initial film thickness is small is closer to the contact than Eq. (11).

In the outlet region, if $X$ is relatively small, Eqs. (10) and (11) agree well with the experimental results. As $X$ increases, the angle between the real cavity boundary and the $X$ axis decreases, and similarly the outer meniscus deviates outward from Eq. (11). The cavity boundary and the outer meniscus become nearly parallel at a downstream location and the side bands are formed between the two. The position of the center of each band is in a good agreement with the intersection point of Eqs. (10) and (11).

Figures 7-12 show that the side bands move away from the center of the track as the speed increases. This can be explained as follows. $\theta^{\prime}$ increases with the speed as seen in Fig. 1, while $\theta\left(X_{0}, Y\right) H\left(X_{0}, Y\right)$, the film thickness in the cavity is approximately of the Hertzian profile and thus hardly affected by the speed. This means that, according to Eq. $(10), H(X, Y)$ at the film rupture point decreases and hence the cavity boundary moves upstream as the speed increases. Since the outer meniscus is also hardly affected by the speed, the intersection point of Eqs. (10) and (11) moves away from the center of the track. That is, the side bands move outward as the speed increases.

By comparing between Figs. 10-12, we can see that the dimensionless inlet distance increases with the dimensionless maximum film thickness of the side bands, which affects the amount of oil replenished from the side

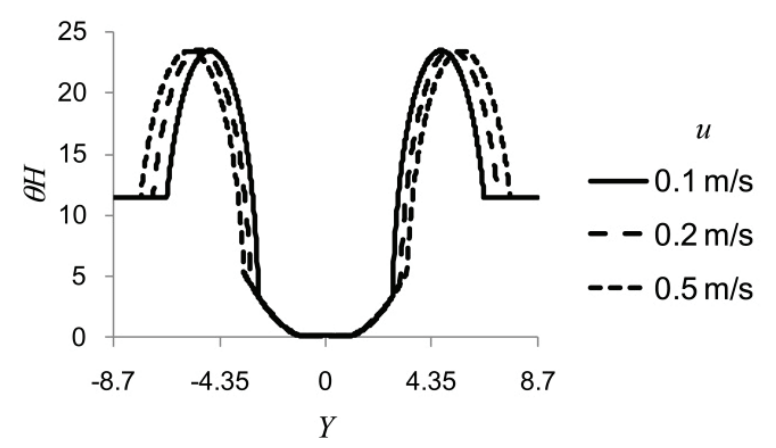

Fig. 7 Dimensionless inlet film thickness (\#2)

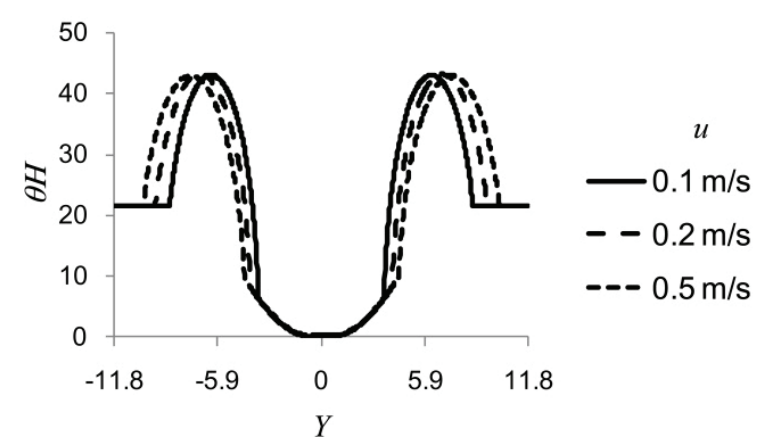

Fig. 8 Dimensionless inlet film thickness (\#3)

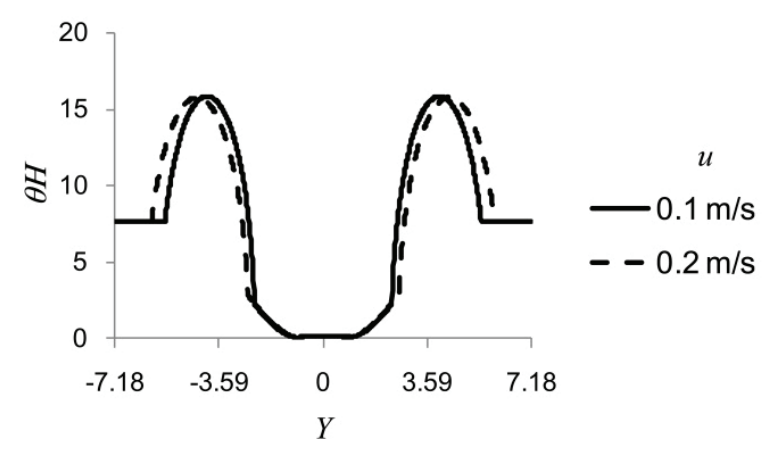

Fig. 9 Dimensionless inlet film thickness (\#4) 


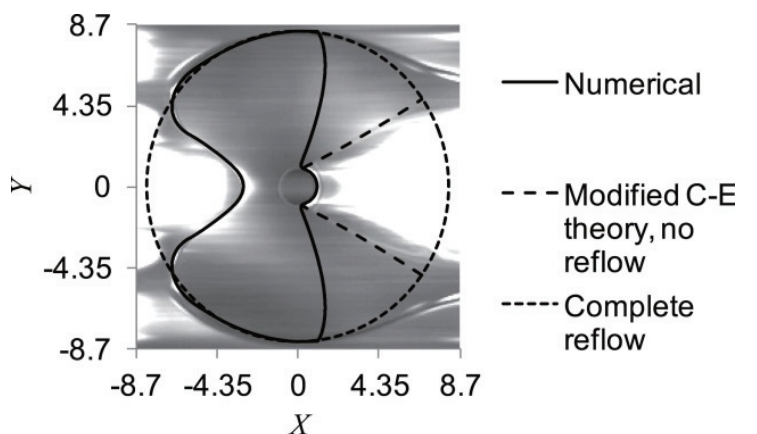

(a) $u=0.1 \mathrm{~m} / \mathrm{s}$

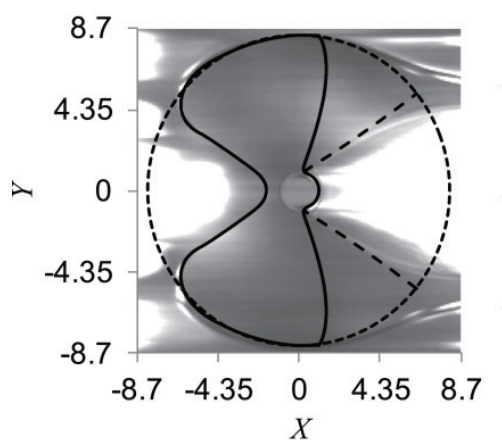

(b) $u=0.2 \mathrm{~m} / \mathrm{s}$

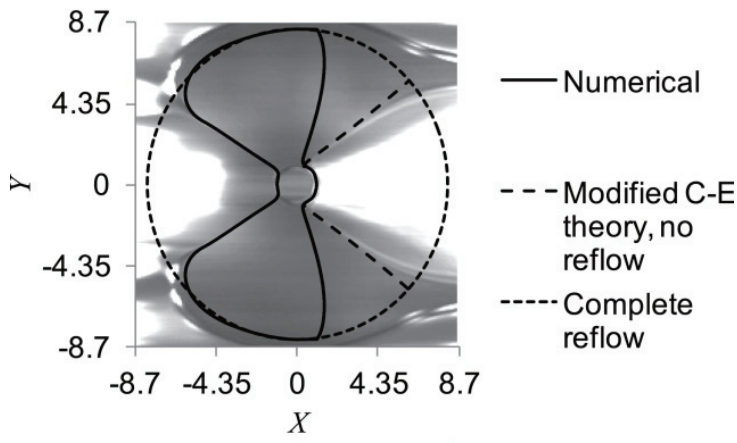

(c) $u=0.5 \mathrm{~m} / \mathrm{s}$

Fig. 10 Analytical results and photographs of the meniscus (\#2)

bands to the inlet. Therefore, as the speed increases and the side bands move outward, the amount of oil replenished to the inlet decreases and consequently the dimensionless inlet distance decreases. This is why starvation is likely to occur at higher speeds. A large difference in the inlet distance is caused by a small difference in the position of the side bands. This is because most of the oil exists in the side bands and the amount of oil at the center of the track is extremely small.

The oil tested in Figs. 10-12 was paraffinic mineral, but synthetic hydrocarbon and perfluoropolyether oils were also tested as mentioned in the chapter 3 . The test results showed that the meniscus shape is mainly determined by the dimensionless initial film thickness and the capillary number regardless of the type of oil. For example, the meniscus shape is almost identical between the case of $u=0.5 \mathrm{~m} / \mathrm{s}$ in \#1 (paraffinic mineral oil, $H_{i}=$ 20 and $C=1.6$ ) and the case of $u=0.2 \mathrm{~m} / \mathrm{s}$ in \#5

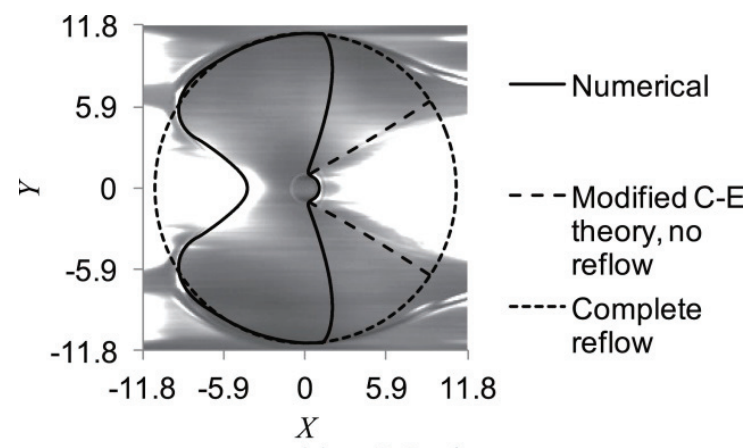

(a) $u=0.1 \mathrm{~m} / \mathrm{s}$

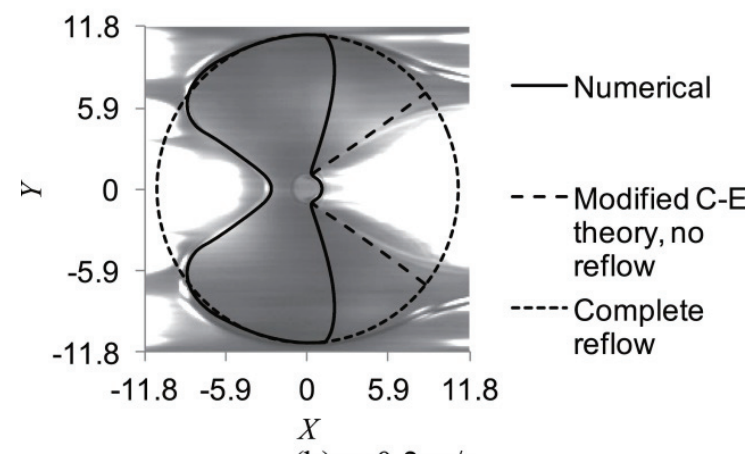

(b) $u=0.2 \mathrm{~m} / \mathrm{s}$

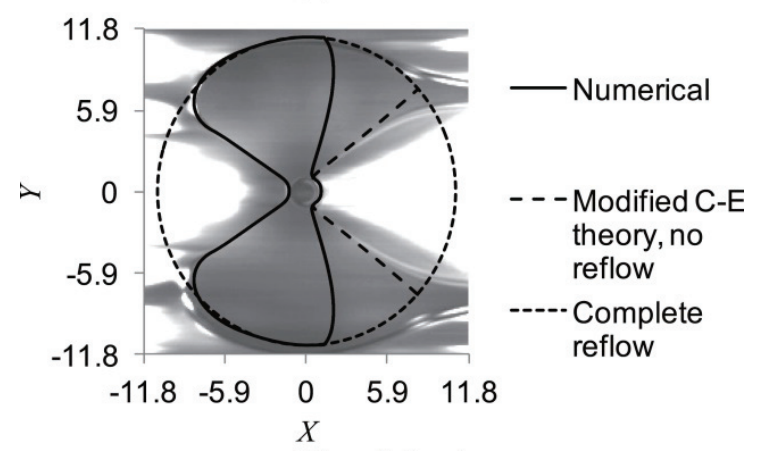

(c) $u=0.5 \mathrm{~m} / \mathrm{s}$

Fig. 11 Analytical results and photographs of the meniscus (\#3)

(synthetic hydrocarbon oil, $H_{i}=20$ and $C=1.6$ ). Therefore, in the following comparison between the analytical results and the experimental results for all the oils tested, we focus on the effect of $H_{i}$ and $C$.

Figure 13 shows the relation between the dimensionless inlet distance at the center of the track and the capillary number, where the diameter of bubbles is proportional to the dimensionless initial film thickness. The analytical results and the experimental results are in a good agreement. As the capillary number increases, the dimensionless inlet distance decreases, while it increases with the diameter of the bubbles, i.e. the dimensionless initial film thickness. For the results shown in Fig. 13, six parameters: surface tension, viscosity, viscosity-pressure coefficient, speed, load and the initial film thickness were varied. However, we can see that the dimensionless inlet distance is mainly determined by the two parameters, $H_{i}$ and $C$. 


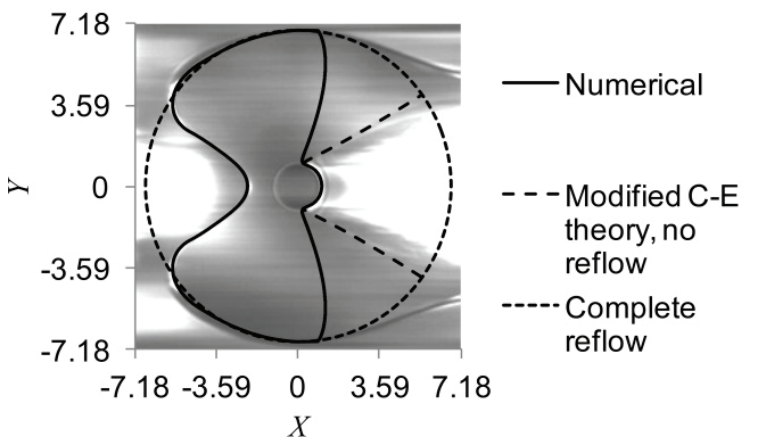

(a) $u=0.1 \mathrm{~m} / \mathrm{s}$

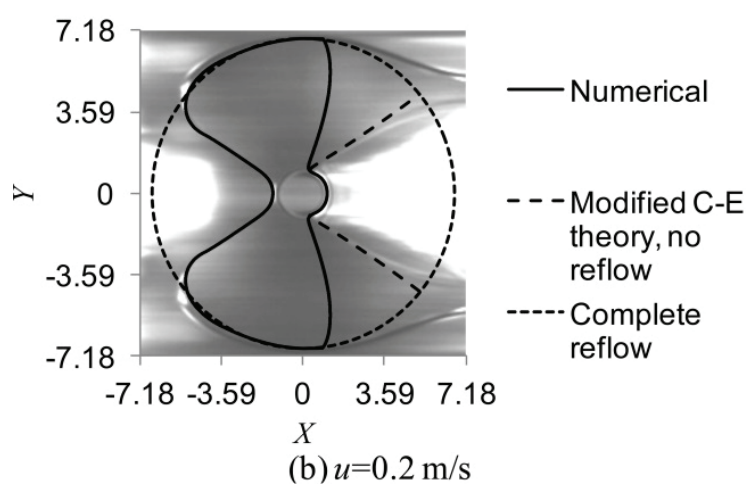

Fig. 12 Analytical results and photographs of the meniscus (\#4)

Figure 14 shows $H_{c} / H_{c f f}$, the reduction factor of the central film thickness, namely, the ratio of the starved central film thickness to the fully flooded central film thickness, as a function of a parameter $(m-1) /\left(m^{*}-1\right)$ proposed by H-D. $m^{*}$ for the central film thickness is given by

$$
m^{*}=1+3.06 H_{c f f}^{0.58}
$$

Figure 14 also shows the H-D formula for the reduction factor of the central film thickness by a solid line.

$$
\frac{H_{c}}{H_{c f f}}=\left(\frac{m-1}{m^{*}-1}\right)^{0.29}
$$

The analytical results and the experimental results are in a good agreement. A good correlation is obtained between Eq. (19) and the present results, which confirms that the reduction factor of the central film thickness is determined by the dimensionless inlet distance. In the scope of this paper, $H_{c f f}$ obtained with the numerical analysis agrees well with the fully flooded H-D formula. We used the former to calculate the reduction factor of the central film thickness in the numerical results and the latter to calculate $m^{*}$.

The case of $u=0.5 \mathrm{~m} / \mathrm{s}$ in $\# 2$ resulted in $m \approx 1$, i.e. the inlet meniscus approximately coinciding with the Hertzian circle, and the iteration of the EHL computation and the inlet film thickness calculation was terminated after 10 steps, since the solution did not converge. In this condition, the oil is not replenished to the inner part of the track and the film thickness gradually decreases due

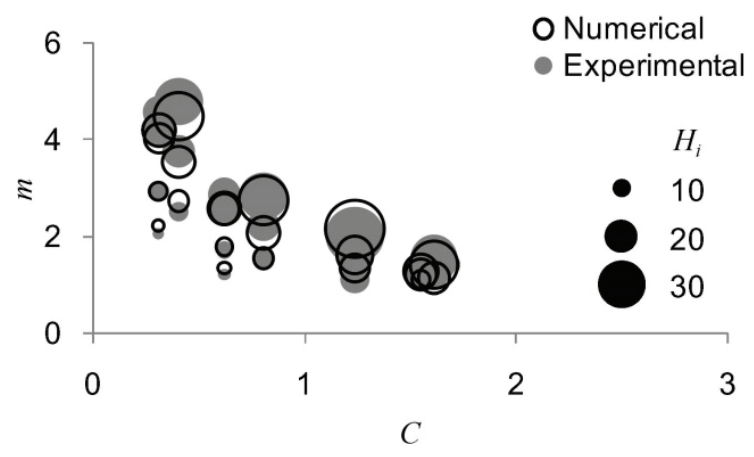

Fig. 13 Dimensionless inlet distance

to side leakage. Similar results are obtained by using the outlet film thickness having passed through the contact as the inlet film thickness [3]. In this case, theoretically, the film thickness monotonically decreases and the asperity contact between two surfaces is supposed to be inevitable after repeated overrollings. However, some earlier studies reported the existence of an extremely thin oil film even under severely starved conditions [7]. In rolling bearings, given that the amount of oil on the track may change due to ball/cage contacts, the lubrication mechanism is even more complicated.

\section{Conclusions}

Based on the Coyne-Elrod theory which takes surface tension into account, a method was proposed to calculate the nonuniform inlet film thickness including side bands. By the Elrod algorithm which automatically determines the meniscus using the inlet film thickness as a boundary condition, starved EHL point contacts were analyzed. Results for the meniscus and the film thickness were presented and validated with the experimental results. The following conclusions were drawn.

(1) In the inner part of the track, the cavitation boundary is approximately straight and extends obliquely downstream from the Hertzian circle, and the angle between the boundary and the $X$ axis increases as the capillary number increases. On the other hand, the meniscus on both sides of the track is elliptical and

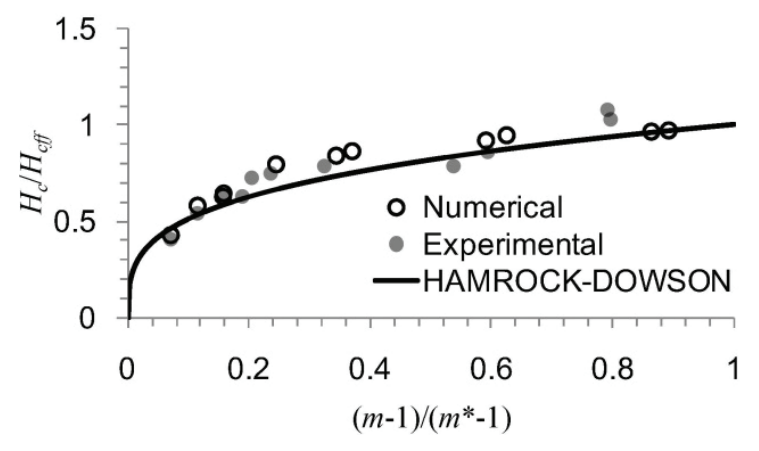

Fig. 14 Film thickness reduction 
mostly determined by the amount of oil, i.e. the dimensionless initial film thickness. The side bands are formed at the intersection of the cavity boundary and the outer meniscus. Therefore, the side bands move away from the center of the track as the capillary number increases.

(2) The dimensionless inlet distance strongly depends on the amount of oil replenished from the side bands to the inlet, and increases as the dimensionless initial film thickness increases. As the capillary number increases and the side bands move outward, the amount of oil replenished from the side bands to the inlet decreases and thus the dimensionless inlet distance decreases. This is the mechanism of starvation in EHL point contacts under high speeds.

(3) The analytical results and the experimental results are in a good agreement for the shape of the meniscus and hence the dimensionless inlet distance. The dimensionless inlet distance is mainly determined by two parameters: the dimensionless initial film thickness and the capillary number. The analytical results and the experimental results are also in a good agreement for the reduction factor of the central film thickness, which is well correlated with the parameter $(m-1) /\left(m^{*}-1\right)$ proposed by H-D.

\section{Nomenclature}

a Hertzian radius

C Capillary number

$h \quad$ Film thickness

$h_{c} \quad$ Central film thickness

$h_{c f f} \quad$ Fully flooded central film thickness

$h_{i} \quad$ Initial film thickness

$H$ Dimensionless film thickness, $H=h R_{x} / a^{2}$

$H_{c}$ Dimensionless central film thickness, $H_{c}=$ $h_{c} R_{x} / a^{2}$

$H_{c f f}$ Dimensionless fully flooded central film thickness, $H_{c f f}=h_{c f f} R_{x} / a^{2}$

$H_{e} \quad$ Dimensionless outlet film thickness without side bands

$H_{i} \quad$ Dimensionless initial film thickness, $H_{i}=h_{i} R_{x}$ $/ a^{2}$

$H_{\text {oil }} \quad$ Dimensionless inlet film thickness

$H_{s b} \quad$ Dimensionless maximum film thickness of the side bands

$H_{0} \quad$ Dimensionless rigid body displacement

$m$ Dimensionless inlet distance

$p \quad$ Pressure

$p_{h} \quad$ Maximum Hertzian pressure

$p_{r} \quad$ Constant in the Roelands equation, $p_{r}=1.96 \times$ $10^{8}$

$P \quad$ Dimensionless pressure, $P=p / p_{h}$

$q \quad$ Flow rate per unit width

$Q$ Dimensionless flow rate per unit width, $Q=$ $q R_{x} / u a^{2}$

$r_{x}, r_{y} \quad$ Radii of the meniscus

$r_{X}, r_{Y}$ Dimensionless radii of the meniscus, $r_{X}=r_{x} / a$,

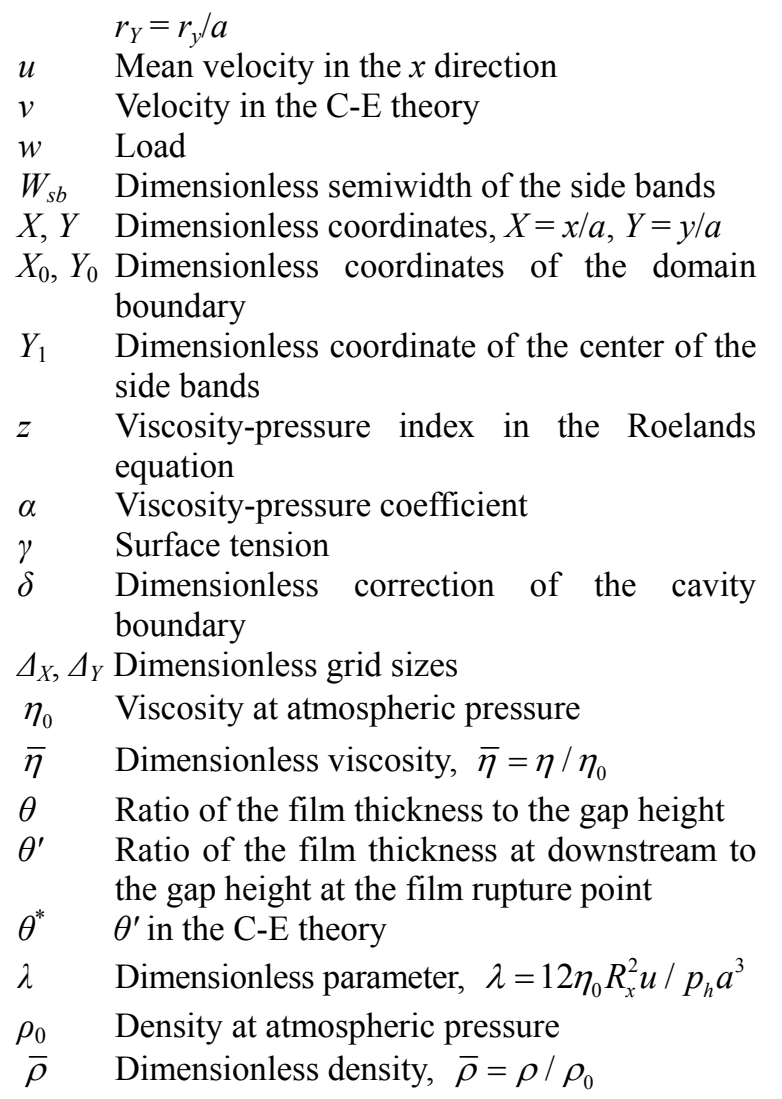

\section{References}

[1] Wedeven, L. D., Evans, D. and Cameron, A., "Optical Analysis of Ball Bearing Starvation," ASME Journal of Lubrication Technology, 93, 3, 1971, 349-361.

[2] Hamrock, B. J. and Dowson, D., "Isothermal Elastohydrodynamic Lubrication of Point Contacts, Part IV-Starvation Results," ASME Journal of Lubrication Technology, 99, 1, 1977, 15-23.

[3] Chevalier, F., Lubrecht, A. A., Cann, P. M. E., Colin, F. and Dalmaz, G., "Film Thickness in Starved EHL Point Contacts," ASME Journal of Tribology, 120, 1, 1998, 126-133.

[4] Elrod, Jr., H. G., "A Cavitation Algorithm,” ASME Journal of Lubrication Technology, 103, 3, 1981, 350-354.

[5] Kingsbury, E., "Cross Flow in a Starved EHD Contact," ASLE Transactions, 16, 4, 1973, 276-280.

[6] Chiu, Y. P., "An Analysis and Prediction of Lubricant Film Starvation in Rolling Contact Systems," ASLE Transactions, 17, 1, 1974, 22-35.

[7] Cann, P. M. E., Damiens, B. and Lubrecht, A. A., "The Transition between Fully Flooded and Starved Regimes in EHL," Tribology International, 37, 10, 2004, 859-864.

[8] Shibasaki, K., Taniguchi, M. and Oshima, M., "Development of Numerical Method for Coupled Simulation of Starved EHL and Macro Flow," 
Report of the Institute of Industrial Science, University of Tokyo, 62, 1, 2010, 40-44 (in Japanese).

[9] Shibasaki, K., Maruyama, T. and Oshima, M., "Coupled Simulation of Starved EHL and Macro Flow on Ball-on-Disc, and Experimental Validation," Proceedings of JAST Tribology Conference, Tokyo, May 2011, 267-268 (in Japanese).

[10] Pemberton, J. and Cameron, A., "A Mechanism of Fluid Replenishment in Elastohydrodynamic Contacts," Wear, 37, 1, 1976, 185-190.

[11] Wijnant, Y. H., "Contact Dynamics in the Field of Elastohydrodynamic Lubrication," Ph. D. thesis, University of Twente, 1998.

[12] Coyne, J. C. and Elrod, Jr., H. G., "Conditions for the Rupture of a Lubricating Film, Part I: Theoretical Model," ASME Journal of Lubrication Technology, 92, 3, 1970, 451-456.

[13] Coyne, J. C. and Elrod, Jr., H. G., "Conditions for the Rupture of a Lubricating Film, Part II: New Boundary Conditions for Reynolds Equation," ASME Journal of Lubrication Technology, 93, 1, 1971, 156-167.

[14] Nogi, T. and Kato, T., "Influence of a Hard Surface Layer on the Limit of Elastic Contact (Part 1)-Analysis Using a Real Surface Model-," Journal of Japanese Society of Tribologists, 42, 2, 1997, 158-165 (in Japanese).

[15] Hewson, R. W., "Free Surface Model Derived From the Analytical Solution of Stokes Flow in a Wedge," ASME Journal of Fluids Engineering, 131, 4, 2009, 041205-1-041205-5.

[16] Dalmaz, G., "Formation and Separation of Thin Viscous Film in Hertzian Line Contacts," ASME Journal of Lubrication Technology, 102, 4, 1980, 466-477.

[17] Birkhoff, G. and Hays, D. F., "Free Boundaries in Partial Lubrication," Journal of Mathematics and Physics, 42, 2, 1963, 126-138.

[18] Popovici, G., "Effects of Lubricant Starvation on Performance of Elast-Hydrodynamically Lubricated Contacts," Ph. D. Thesis, University of Twente, 2005.

[19] Nakahara, T., "Fluid Film Behavior-Cavitation-," Journal of Japanese Society of Tribologists, 26, 3, 1981, 146-152 (in Japanese).

\section{Appendix A: Calculation method of the C-E boundary condition}

The C-E theory is modified to avoid numerical instability. Only in the Appendix A, notations and numbers of equations are matched those in [12]. Let the free surface velocity component normal to the boundary $w=0$ in Eq. (3).

$$
\left(\frac{\partial u}{\partial y}\right)_{y=l}=0
$$

From Eq. (23),

$$
V=\frac{3}{2} \cos \theta\left(\frac{1}{H}-\frac{1}{3}\right)
$$

Substituting the above equation into Eq. (27),

$$
\frac{d \theta}{d S}=\frac{\psi+\frac{R}{6}\left(V^{2}-1\right)+G(H-1)-\frac{3 \sin \theta \cos \theta}{2 H^{2}}}{\frac{1}{N^{3}}+V \sin \theta}
$$

Equation (27) is erroneous and the 3rd term should be $-G(H-1)$. A numerical integration of the above Eqs. (A1), (A2), (26), (28) and (29) gives the solution. In this numerical integration, there is no instability problem pointed out in [15]. Linearization in the same way as C-E modifies Eq. (37) as

$$
\psi=\frac{A \exp (\lambda S)}{\lambda}
$$

and Eq. (40) as

$$
\frac{1}{N^{3}} \lambda^{3}+\frac{3}{2} \lambda^{2}+\left(\frac{R}{2}-G\right) \lambda-1=0
$$

The above Eqs. (A4), (A5), (35), (36) and (39) give initial values for the numerical integration. Table A1 shows a comparison between the tabulated values given in [12] (in the case of $R=G=0$ ) and the above method. We can see that the difference in $c / h_{\infty}$ is small, which is the reciprocal of $\theta^{*}$ in the text.

Table A1 Comparison of the present theory with the C-E theory

\begin{tabular}{c|c|c|c|c}
\hline \multirow{2}{*}{$\begin{array}{c}N= \\
(3 C)^{1 / 3}\end{array}$} & \multicolumn{2}{|c|}{ C-E theory } & \multicolumn{2}{c}{ Present theory } \\
\cline { 2 - 5 } & $c / h_{\infty}$ & $R_{0} / h_{\infty}$ & $c / h_{\infty}$ & $R_{0} / h_{\infty}$ \\
\hline 0.2 & 43.2 & 41.6 & 42.9 & 40.7 \\
\hline 0.5 & 9.18 & 7.37 & 8.95 & 6.87 \\
\hline 1.0 & 3.84 & 1.88 & 3.72 & 1.58 \\
\hline 1.5 & 2.84 & 0.717 & 2.82 & 0.722 \\
\hline 2.0 & 2.52 & 0.331 & 2.56 & 0.506 \\
\hline 5.0 & 2.28 & 0.023 & 2.37 & 0.364 \\
\hline
\end{tabular}

\section{Appendix B: C-E Boundary condition in rolling contacts}

From the experimental results by Dalmaz [16], we obtain the ratio of the film thickness at downstream to the gap height at the film rupture point. In rolling line 
contacts, according to Birkhoff and Hays [17],

$$
h_{\infty} / h_{\min }=0.65
$$

where $h_{\min }$ is the minimum film thickness and we assume that $h_{\infty}$ is equal on two surfaces. $2 h_{s}$, the gap height at the film rupture point is given by

$$
2 h_{s}=h_{\text {min }}+\frac{x_{s}^{2}}{2 R_{x}}
$$

where $x_{s}$ is the position of the film rupture point. Therefore,

$$
\bar{x}_{s}=\frac{x_{s}}{\sqrt{2 R_{x} h_{\min }}}=\sqrt{\frac{1.3}{\theta^{\prime}}-1}
$$

Figure B1 shows $\theta^{\prime}$ obtained from the experimental results of $\bar{x}_{s}$ and Eq. (B3). Assuming $\theta^{\prime}$ is proportional to $\theta^{*}$, Eq. (8) is obtained by the least squares method. Equation (8) and the experimental results are in a good agreement.

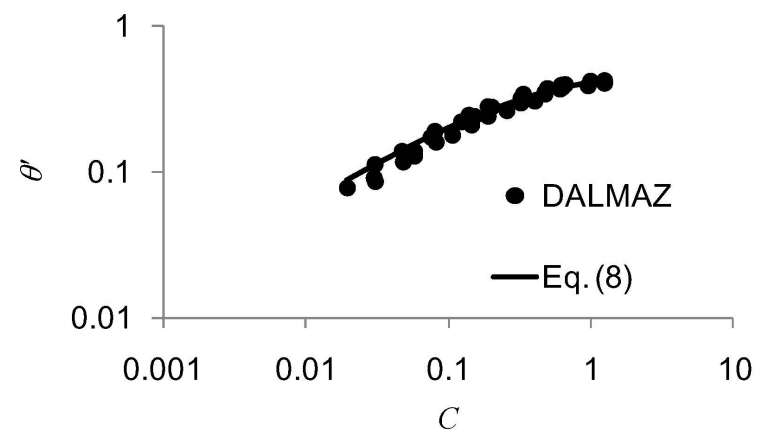

Fig. B1 Ratio of the film thickness downstream to the point of film rupture 